\title{
Erratum zu „Mikrofluidischer Chip vereint QCM-D und mikroskopische Messungen“
}

ADAM L. J. OLSSON, PAULA BRAUN, JIN ZHANG, HARTMUT DRECHSEL, ALBERT STERCK, GUDRUN PREPENS, THOMAS REINER, FRANK K. GEHRING

3T GMBH \& CO KG, TUTTLINGEN

DOI: $10.1007 / \mathrm{s} 12268-019-0195-\mathrm{y}$ (C) Springer-Verlag 2019

Veröffentlicht: Mai 2019

(C) Springer-Verlag 2019

DOI: 10.1007/s12268-019-1051-9

Auf Wunsch der Autoren werden folgende Personen zusätzlich als Autoren gelistet:
Zeqian Xu, Universitätsklinikum Tübingen, Sektion Medizinische Werkstoffkunde \& Technologie, Tübingen;

Stefanie Krajewski, Universitätsklinik für Thorax-, Herz- und Gefäßchirurgie, Klinisches Forschungslabor, Tübingen und

Frank Rupp, Universitätsklinikum Tübingen, Sektion Medizinische Werkstoffkunde \& Technologie, Tübingen.
Außerdem wurde die Danksagung von den Autoren umgeschrieben. Unten finden Sie den korrigierten Artikelkopf und die neue Danksagung.

Die Online-Version des Originalartikels finden Sie unter https://link.springer.com/ article/10.1007/s12268-019-1051-9.

\section{Lab-on-a-chip}

\section{Mikrofluidischer Chip vereint QCM-D und mikroskopische Messungen}

ADAM L. J. OLSSON ${ }^{1}$, ZEQIAN XU ${ }^{2}$, PAULA BRAUN ${ }^{1}$, JIN ZHANG ${ }^{1}$, HARTMUT DRECHSEL ${ }^{1}$, ALBERT STERCK ${ }^{1}$, GUDRUN PREPENS ${ }^{1}$, THOMAS REINER ${ }^{1}$, STEFANIE KRAJEWSKI ${ }^{3}$, FRANK RUPP ${ }^{2}$, FRANK K. GEHRING ${ }^{1}$

$13 \mathrm{~T} \mathrm{GMBH} \& \mathrm{CO} \mathrm{KG}$, TUTTLINGEN

${ }^{2}$ UNIVERSITÄTSKLINIKUM TÜBINGEN, SEKTION MEDIZINISCHE WERKSTOFFKUNDE \& TECHNOLOGIE, TÜBINGEN

${ }^{3}$ UNIVERSITÄTSKLINIK FÜR THORAX-, HERZ- UND GEFÄßCHIRURGIE, KLINISCHES FORSCHUNGSLABOR, TÜBINGEN

\section{Danksagung}

Diese Studie wurde gefördert durch AiF ZIM (KF2806411CR4). Zeqian Xu wird durch den China Scholarship Council (201708080003) gefördert.

Korrespondenzadresse:

Dr. Frank K. Gehring

3T analytik

Gartenstraße 100

D-78532 Tuttlingen

Tel.: 07461-165030

info@3t-analytik.de

www.3t-analytik.de 